\title{
Efecto de la siembra directa sobre los procesos de adsorción de fósforo en los molisoles del centro norte de la provincia de Córdoba (Argentina)
}

\author{
Rollán, A. A. del C., Bachmeier, O. A., Silva Rossi, M. M. y Moreno, M. A.
}

\begin{abstract}
RESUMEN
Bajo la hipótesis que señala que en condiciones de siembra directa (SD) la estratificación de las propiedades edáficas modifica los parámetros de sorción de fósforo $(P)$, se plantearon como objetivos de este trabajo: i) estimar los parámetros de sorción $\mathrm{P}$ mediante uso de isotermas de Langmuir en muestras de distinta profundidad provenientes de lotes de cultivo bajo SD continua; ii) analizar las similitudes y diferencias entre los parámetros obtenidos; y iii) identificar la relación entre las propiedades edáficas del estrato superficial y los parámetros estimados. Se equilibraron muestras de suelo de 0 a 5 y 0 a 20 $\mathrm{cm}$ con soluciones de concentración creciente en $\mathrm{P}$. A partir de la relación entre el $\mathrm{P}$ adsorbido y la concentración de $\mathrm{P}$ en equilibrio se estimó la capacidad máxima de adsorción $\left(Q_{\max }\right)$ y la constante de afinidad de retención $(k)$. El mayor $Q_{\max }$ y la baja $k$ de las muestras de 0 a $5 \mathrm{~cm}$ demuestran que existe un comportamiento diferencial de los primeros centímetros de profundidad. En ese espesor la biodisponibilidad de P y la residualidad de los fertilizantes aplicados están reguladas por fenómenos de adsorción por parte de los coloides orgánicos, acumulados por el proceso de su estratificación asociado a la SD.
\end{abstract}

Palabras clave: Langmuir, estratificación, materia orgánica del suelo, Haplustoles.

Rollán, A. A. del C., Bachmeier, O. A., Silva Rossi, M. M. and Moreno, M. A., P. 2017. Effect of direct seeding on phosphorus adsorption processes in the Mollisols of the center-north of the province of Córdoba (Argentina). Agriscientia 34 (II): 1-11

\section{SUMMARY}

Under the hypothesis that the stratification of soil properties under direct seeding conditions (SD) modifies P sorption parameters, the objectives of this work were: i) to estimate $\mathrm{P}$ sorption parameters by using Langmuir isotherms in samples of different depth, extracted from fields under continuous direct seeding; (ii) to analyze the similarities and differences between the parameters obtained; and (iii) to identify the relationship between the edaphic properties of the surface stratum and the estimated parameters. Soil samples of 0 to 5 and 
0 to $20 \mathrm{~cm}$ were equilibrated with solutions with increasing concentrations of $P$. From the relationship between the adsorbed $P$ and the concentration of $P$ in equilibrium, the maximum adsorption capacity $\left(Q_{m a x}\right)$ and the retention affinity constant $(k)$ were estimated. The higher $Q_{\max }$ and the low $k$ of 0 to $5 \mathrm{~cm}$ samples show that there is a distinct behaviour in the first centimeters of depth. In that layer the bioavailability of $P$ and the residuality of the applied fertilizers are regulated by adsorption phenomena responding to the presence of organic colloids, which are accumulated by the stratification associated with SD.

Key words: Langmuir, soil stratification, soil organic matter, Haplustolls.

Rollán, A. A. del C., Moreno, M. A. y Bachmeier, O. A.: Facultad de Ciencias Agropecuarias, Universidad Nacional de Córdoba, Av. Valparaíso s/n, C.C. 509, 5000, Córdoba, Argentina. Silva Rossi, M. M.: Estudio Agronómico, Venado Tuerto, Argentina. Correspondencia a: arollan@agro.unc.edu.ar

\section{INTRODUCCIÓN}

En Argentina la deficiencia de fósforo en los suelos del trópico y subtrópico es un problema de reconocida importancia (Vázquez, Morales, Fernández López y Dalurzo, 2011; Fernández Lopez y Mendoza, 2013); no obstante, el estudio de esta problemática no se restringe solo a estos ambientes. Los bajos niveles de P disponibles son la causa de la restricción del rendimiento en distintas regiones agrícolas del país (Sainz Rozas, Echeverría y Angelini, 2012).

El origen de las deficiencias de $\mathrm{P}$ puede ser genético o bien el resultado del deterioro físico y químico de los suelos, situación que resulta tanto más grave al momento de evaluar la biodisponibilidad del P edáfico para las plantas (Yadav y Verma, 2012). La actividad humana afecta el ciclo de los nutrientes y, en los agroecosistemas, los cambios en las prácticas de manejo inciden sobre la disponibilidad del nutriente (Richardson, Condron y Haygarth, 2015; Pose, Baeza, Zamuner, Di Gerónimo y Videla, 2016; Moreira et al., 2016).

En Argentina el cambio abrupto de tecnología, dado por la adopción de la siembra directa (SD), tuvo un marcado impacto en la provincia de Córdoba, tanto en la superficie sembrada como en la producción por unidad de superficie, acentuando la presión extractiva sobre el recurso suelo (Sánchez y Barberis, 2013; Schmidt y Amiotti, 2015).

En la actualidad, los suelos franco-limosos del área centro-norte de la provincia de Córdoba muestran cambios físicos y funcionales como resultado del deterioro generado por la SD continua (Rollán y Bachmeier, 2015). A la naturaleza inestable de estos suelos, debido a su alto contenido de limo (Sánchez y Barberis, 2013), se suma su deterioro químico por la falta de fertilización en condiciones de monocultivo de soja (Rollán, 2016).

Varios autores han estudiado la pérdida de la fertilidad del suelo en SD, como consecuencia de balances consistentemente negativos en la disponibilidad de la mayoría de los nutrientes. Ron et al. (2011) registraron pérdidas de $43 \mathrm{~kg} \mathrm{P} \mathrm{ha}^{-1}$ en lotes bajo SD y destacaron que el $84 \%$ de la masa de nutriente perdida proviene de los primeros seis centímetros de profundidad.

Para generar estrategias de gestión, manejo y aplicación de fertilizantes a fin de mejorar la biodisponibilidad de $\mathrm{P}$ para los cultivos, es necesario una mejor comprensión del ciclo del P en el suelo bajo condiciones ambientales específicas (Shi, Ziadi, Messiga, Lalande y Hu, 2015; Stutte et al., 2015).

Entre los procesos que regulan los niveles de $\mathrm{P}$ presentes en la solución del suelo, el de sorcióndesorción es el más rápido. Por ello, resulta determinante para mantener el equilibrio de iones fosfatos en la solución del suelo, ante la modificación de su concentración desde o hacia otros reservorios (Afif Khouri et al., 2007).

Existe una gran variedad de modelos de equilibrio que permiten el estudio de los procesos de sorción-desorción (Kruse et al., 2015). Autores tales como Al Salama (2008), Silva Rossi, Rollan y Bachmeier (2013), Moreira et al. (2016), Pose et al. (2016), entre otros, concluyen que el ajuste de los datos experimentales al modelo de Langmuir (Sparks, 1995) permite evaluar adecuadamente la dinámica del $\mathrm{P}$ en una amplia gama de suelos.

Existe suficiente evidencia experimental que avala el concepto que los cambios inducidos por 
la SD se reflejan principalmente en los primeros centímetros de profundidad (Álvarez et al., 2011; Rollán y Bachmeier, 2015; Schmidt y Amiotti, 2015). Sin embargo, la información referida a la profundidad de muestreo para la evaluación del P en SD es contradictoria (Calviño, Echeverria y Redolatti, 2000).

La mayoría de los trabajos que estudian los fenómenos de sorción de $\mathrm{P}$ lo hacen en muestras de suelo de 0 a $20 \mathrm{~cm}$ de profundidad, aun si provienen de lotes bajo SD (Vázquez y Morales, 2000; Moazed, Hoseini, Naseri y Abbasi, 2010; Chimdi, Gebrekidan, Tadesse y Kibret, 2013; Silva Rossi et al., 2013). Por otro lado, reportes recientes remarcan la importancia de estudiar la dinámica del $\mathrm{P}$ en los primeros centímetros de profundidad (Pose et al., 2016).

Bajo la hipótesis que señala que en condiciones de SD la estratificación superficial de las propiedades edáficas modifica los parámetros de sorción de $\mathrm{P}$ y genera un comportamiento diferencial según la profundidad de muestreo, se plantearon los siguientes objetivos para este trabajo: i) estimar los parámetros de sorción $\mathrm{P}$ mediante el uso de isotermas de Langmuir en muestras de distinta profundidad, provenientes de lotes de cultivo bajo SD continua; ii) analizar el impacto de la estratificación inducida por la SD sobre los valores obtenidos; y iii) identificar la relación entre las propiedades edáficas y los parámetros de sorción estimados.

\section{MATERIALES Y MÉTODOS}

La investigación se realizó en el Laboratorio de Suelos y Aguas (LabSA) de la Facultad de Ciencias Agropecuarias en la Universidad Nacional de Córdoba (Argentina). Se trabajó con muestras de suelo provenientes de lotes de producción bajo agricultura continua en condiciones de SD.

\section{Características del área de estudio}

El área de estudio se ubica dentro de la zona X, de acuerdo con la delimitación de zonas agroeconómicas homogéneas elaborada por Ghida Daza y Sánchez (2009): zona agrícola ganadera del centro de la provincia de Córdoba. Incluye las subzonas X-B Río Primero: semiárida del centro norte, y la subzona X-B Oncativo: semiárida central.

Los Haplustoles (H. énticos y H. típicos), son los suelos dominantes de la región; se caracterizan por ser suelos productivos, profundos, bien drenados, fértiles, con un horizonte superficial con más del $1 \%$ de materia orgánica y con el complejo de cambio dominado por el calcio, lo cual favorece, junto con el aporte de residuos que realizó la vegetación que compuso el "espinal" original, el desarrollo de una buena estructura. Sin embargo, el alto contenido en limo les confiere cierta fragilidad e inestabilidad estructural, que se manifiesta por una tendencia al encostramiento y al "planchado", punto inicial de los escurrimientos y de los procesos erosivos (Sánchez y Barberis, 2013).

Esta región está profundamente modificada por las actividades agropecuarias, con una casi total sustitución de la vegetación natural (Espinal) por cultivos, primero de trigo, luego de maíz y más recientemente soja (INTA, 2006).

\section{Sitios de muestreo}

En lotes de producción bajo agricultura continua en condiciones de SD, se delimitaron parcelas de $500 \mathrm{~m}^{2}$ que se identificaron como sitios de muestreo (S). Los sitios de muestreo se ubicaron: $\mathrm{S}_{1}$ a $31^{\circ} 18^{\prime} 46^{\prime \prime} \mathrm{S}, 63^{\circ} 52^{\prime} 36^{\prime \prime} \mathrm{O}$ (Montecristo); $\mathrm{S}_{2}$ a $31^{\circ} 18^{\prime} 11^{\prime \prime} \mathrm{S}, 63^{\circ} 43^{\prime} 41^{\prime \prime} \mathrm{O}$ (Piquillín); $\mathrm{S}_{3}$ a $31^{\circ} 43^{\prime}$ $37^{\prime \prime} \mathrm{S}, 63^{\circ} 55^{\prime} 53^{\prime \prime} \mathrm{O}$ (Pilar); $\mathrm{S}_{4}$ a $30^{\circ} 36^{\prime} 43^{\prime \prime} \mathrm{S}, 64^{\circ}$ 13'49" O (Avellaneda); $S_{5}$ a $30^{\circ} 55^{\prime} 41^{\prime \prime} \mathrm{S}, 64^{\circ} 12^{\prime}$ $10^{\prime \prime} \mathrm{O}$ (La Paz) y $\mathrm{S}_{6}$ a $30^{\circ} 45^{\prime} 26^{\prime \prime} \mathrm{S}, 63^{\circ} 43^{\prime} 07^{\prime \prime} \mathrm{O}$ (Cañada de Luque).

\section{Muestreo y medidas}

Se tomaron muestras compuestas por veinticinco submuestras de 0 a $5 \mathrm{~cm}$ y 0 a $20 \mathrm{~cm}$ de profundidad, las que fueron secadas, molidas y tamizadas por malla de $2 \mathrm{~mm}$.

Para la caracterización del suelo de los sitios de muestreo se midió: carbono oxidable (COS) por la técnica de Walkley y Black (Nelson y Sommer, 1996), pH 1:2,5 por potenciometría, fósforo extractable (P-Bray) por el método de Bray y Kurtz $N^{\circ}$ 1 , contenido de arcilla $<2 \mu \mathrm{m}$ mediante el método de la pipeta (Soil Conservation Service, 1972), capacidad de intercambio catiónico $(\mathrm{ClC})$ y cantidad de calcio intercambiable $\mathrm{Ca}^{2+}$ por desplazamiento con $\mathrm{NH}_{4} \mathrm{OAc} 1 \mathrm{M}$ a pH 7 y posterior valoración quelatométrica con EDTA 0,01 M. Todas las determinaciones se llevaron a cabo por triplicado.

\section{Tratamientos de adsorción de $\mathrm{P}$ y diseño ex- perimental}

Las unidades experimentales consistieron en recipientes de Nalgene de $50 \mathrm{ml}$ de capacidad que fueron cargados siguiendo la metodología descripta por Bravo, Montoya y Menjivar (2013). El tiempo 
de incubación fue de $96 \mathrm{~h}$ a $25^{\circ} \mathrm{C}$, de acuerdo con Silva Rossi, Rollan y Bachmeier (2016).

Se utilizó un diseño completamente al azar, con tratamientos consistentes en once dosis de $\mathrm{P}$ aplicado en soluciones de $\mathrm{KH}_{2} \mathrm{PO}_{4}(0 ; 1 ; 3 ; 5 ; 7,5 ; 10$; 15; 20; 25; 30 y $\left.40 \mathrm{mg} \mathrm{L}^{-1}\right)$, seis matrices de suelo (una por sitio) y dos profundidades $(0-5$ y $0-20 \mathrm{~cm}$ ). Se trabajó con tres repeticiones por tratamiento.

\section{Evaluación de la adsorción de fósforo}

La diferencia entre las concentraciones de P suministrado y remanente en la solución de equilibrio fue considerada como P adsorbido. Los datos experimentales se ajustaron al modelo de adsorción de Langmuir:

$$
Q=\frac{Q_{\max } \times k \times C}{(1+k \times C)}
$$

Donde $Q$, es la cantidad de $\mathrm{P}$ adsorbido ( $\mathrm{mg}$ $\left.P \mathrm{~kg}^{-1}\right) ; Q_{\max }\left(\mathrm{mg} \mathrm{P} \mathrm{kg}^{-1}\right)$, es una estimación de la cantidad máxima de fósforo adsorbido, $k\left(\mathrm{~L} \mathrm{mg}^{-1}\right)$ representa la energía de retención y $C\left(\mathrm{mg} \mathrm{L}^{-1}\right)$ la concentración en la solución del suelo en el equilibrio (Sparks, 1995).

\section{Análisis estadístico}

Se realizó mediante regresiones no lineales. Los datos de caracterización de los sitios fueron sometidos al análisis de varianza (ANOVA) y las medias fueron comparadas mediante la prueba LSD-Fisher $(p<0,001)$. En todos los casos se utilizó el programa InfoStat (Di Rienzo et al., 2013).

\section{RESULTADOSY DISCUSIÓN}

\section{Características de la matriz de suelo adsorbente}

Los datos analíticos correspondientes a la caracterización de los sitios de muestreo se presentan en la Tabla 1. Los suelos estudiados presentan bajo contenido de arcilla (227 $\mathrm{g} \mathrm{kg}^{-1}$, en promedio), condición característica de los horizontes A de los Haplustoles del centro de Córdoba, en los que la fracción dominante es la de limo (INTA, 2006). No hubo diferencias significativas en el contenido de arcilla entre sitios ni entre profundidades.

El contenido de arcillas es una propiedad muy importante en la sorción de P; el incremento en la retención de $\mathrm{P}$ está directamente relacionado con el porcentaje de arcillas presente cuando los valores superan el 30 \% (Eriksson, 2016). En este estudio, la falta de diferencias significativas entre sitios y profundidades homogeniza las características mineralógicas de la matriz adsorbente. Además, los valores presentes (menores al $30 \%$ ) condicionan el uso del contenido de arcilla como único atributo del suelo para la recomendación de la fertilización fosfatada, tal como se hace rutinariamente (Pinto, Damacena de Souza, Barbosa Paulino, Curi y Carbone Carneiero, 2013).

El pH en los S varió entre un mínimo de 5,9 y un máximo de 6,9, valores propios de los suelos de la región extrapampeana (Sainz Rozas, Echeverría y Angelini, 2011). Pose et al. (2014) concluyen que, en estas condiciones, con valores de $\mathrm{pH}$ ligeramente ácidos a neutros, la reacción del suelo no resulta determinante en los fenómenos de retención de $\mathrm{P}$, a diferencia de lo que sucede en los suelos ácidos del NE del país (Vázquez y Morales, 2000).

Tabla 1. Características analíticas de los sitios de muestreo (S1 a S6). Valores promedios de: materia orgánica del suelo (MOS), carbono oxidable (COS), contenido de arcilla, $\mathrm{pH} 1: 2,5$, calcio intercambiable $\left(\mathrm{Ca}^{+2}\right)$, capacidad de intercambio catiónico (CIC) y fósforo extractable (P-Bray) medidos en muestras compuestas (x 25) tomadas de 0 a 5 y 0 a $20 \mathrm{~cm}$ de profundidad.

\begin{tabular}{|c|c|c|c|c|c|c|c|c|}
\hline Sitio & Prof.cm & MOS g kg-1 & $\mathrm{COS} \mathrm{g} \mathrm{kg}^{-1}$ & Arcilla g kg-1 & $\mathrm{pH} \mathrm{1:2,5}$ & $\mathrm{Ca}^{+2} \mathrm{cmol}_{\mathrm{c}} \mathrm{kg}^{-1}$ & $\mathrm{ClC} \mathrm{cmol}_{\mathrm{c}} \mathrm{kg}^{-1}$ & P-Bray mg kg-1 \\
\hline \multirow{2}{*}{1} & $0-5$ & $30,0 a$ & $17,7 a$ & $210 a$ & $6,1 a$ & $13,15 a$ & $20,5 a$ & $47,2 \mathrm{a}$ \\
\hline & $0-20$ & $22,8 b$ & $13,2 b$ & $210 a$ & $6,0 a$ & $12,74 a$ & $19,4 a$ & $42,0 a$ \\
\hline \multirow{2}{*}{2} & $0-5$ & $26,9 a$ & $15,6 a$ & $222 a$ & $6,1 a$ & $13,80 a$ & $21,7 a$ & $73,0 b$ \\
\hline & $0-20$ & $20,2 b$ & $11,6 b$ & $222 a$ & $6,1 \mathrm{a}$ & $12,64 a$ & $19,3 a$ & $37,0 a$ \\
\hline \multirow{2}{*}{3} & $0-5$ & $25,6 a$ & $15,6 a$ & $223 a$ & $5,9 a$ & $11,40 a$ & $19,6 a$ & $80,5 c$ \\
\hline & $0-20$ & $20,0 b$ & $11,6 b$ & $223 a$ & $6,1 \mathrm{a}$ & $12,64 a$ & $19,3 a$ & $30,0 a$ \\
\hline \multirow{2}{*}{4} & $0-5$ & $21,8 a$ & $12,6 a$ & $218 a$ & $6,9 a$ & $17,75 b$ & $25,4 b$ & $46,9 a$ \\
\hline & $0-20$ & $19,0 b$ & $11,1 b$ & $218 a$ & $6,6 a$ & $15,69 b$ & $21,1 a$ & $43,0 a$ \\
\hline \multirow{2}{*}{5} & $0-5$ & $40,3 c$ & $24,1 \mathrm{c}$ & $215 a$ & $6,6 a$ & $18,35 b$ & $28,8 b$ & $31,0 a$ \\
\hline & $0-20$ & $31,0 a$ & $18,1 \mathrm{a}$ & $215 a$ & $6,6 a$ & $18,84 b$ & $27,0 b$ & $15,2 d$ \\
\hline \multirow{2}{*}{6} & $0-5$ & $40,2 c$ & $23,3 c$ & $214 a$ & $6,0 a$ & $12,91 a$ & $20,6 a$ & $106,6 e$ \\
\hline & $0-20$ & $28,1 a$ & $16,4 a$ & $214 a$ & $6,1 \mathrm{a}$ & $13,21 a$ & $19,7 a$ & $80,6 f$ \\
\hline
\end{tabular}

Medias con distinta letra indican diferencias estadísticamente significativas en los datos de la columna $(p<0,001)$. 
Los contenidos de COS de 0 a $5 \mathrm{~cm}$ fueron significativamente mayores $(p<0,001)$ a los medidos en las muestras de 0 a $20 \mathrm{~cm}$. Estas diferencias son el resultado de la estratificación superficial de la materia orgánica del suelo (MOS), consecuencia de la SD continua (Álvaro Fuentes, López Sánchez, Cantero-Martínez y Arrúe Ugarte, 2008; Álvarez et al., 2011; Rollán y Bachmeier, 2015; Schmit et al., 2015; Rollán, 2016). Sin embargo, a diferencia de lo observado por Martínez, Fuentes y Acevedo (2008), las diferencias en el COS censadas entre profundidades no impactaron significativamente en el valor de la CIC entre profundidades, pero sí entre S.

En los primeros $5 \mathrm{~cm}$ los valores de $\mathrm{CIC}$ fueron desde un mínimo de $19,6 \mathrm{cmol}_{c} \mathrm{~kg}^{-1}$ a un máximo de $28,8 \mathrm{cmol}_{\mathrm{c}} \mathrm{kg}^{-1}$ con contenidos de COS de 15,6 $\mathrm{mg} \mathrm{kg}^{-1}$ y $24,1 \mathrm{mg} \mathrm{kg}^{-1}$, respectivamente. De 0 a 20 $\mathrm{cm}$ el rango fue de $19,3 \mathrm{cmol}_{\mathrm{c}} \mathrm{kg}^{-1}$ a $27 \mathrm{cmol}_{\mathrm{c}} \mathrm{kg}^{-1}$ para contenidos de COS de $11,6 \mathrm{mg} \mathrm{kg}^{-1}$ y $18,1 \mathrm{mg}$ $\mathrm{kg}^{-1}$, respectivamente.

El suelo bajo estudio presentó un alto grado de saturación con calcio (>50\% ClC). Algunos trabajos atribuyen al calcio y a algunos minerales secundarios, como el carbonato de calcio, un alto grado de participación en los fenómenos de sorción. Carreira, Viñegla y Lajtha (2006) concluyen que en ambientes áridos el principal agente geoquímico capaz de fijar y retener el $\mathrm{P}$ dentro del perfil del suelo es el carbonato de calcio $\left(\mathrm{CaCO}_{3}\right)$. En los suelos evaluados la presencia de $\mathrm{CaCO}_{3}$ en el perfil se observa a partir de los $60 \mathrm{~cm}$ de profundidad estando libre de carbonatos el horizonte superficial (INTA, 2006). Los contenidos de $\mathrm{Ca}^{+2}{ }_{\text {int. }}$ fueron menores a los citados por Carreira et al. (2006) y mayores a los registrados por Rosolem y Merlin (2014), quienes en sus ensayos de fertilización observaron que el $\mathrm{P}$ inorgánico unido a $\mathrm{Ca}$ aumentaba en capas próximas a la superficie del suelo $(0-10 \mathrm{~cm})$ a través de la precipitación secundaria de minerales de fosfato de calcio (Ca-P).

\section{Isotermas de adsorción de P}

En la Tabla 2 se presentan los parámetros de sorción correspondientes a cada sitio y profundidad. Se detallan allí los valores de la cantidad máxima de $\mathrm{P}$ adsorbido $\left(Q_{\max }\right)$ y la energía de retención $(k)$, estimados mediante el ajuste de los datos experimentales al modelo de isoterma de Langmuir.

La contribución más sobresaliente del uso de este tipo de isoterma para la descripción termodinámica de la adsorción de iones fosfato es la consideración de que este fenómeno tiene lugar en un plano imaginario que permite ver la zona de adsorción como una fase (Kruse et al., 2015). Si bien es un modelo simple, la bibliografía remarca la importancia práctica que tiene el ajuste de los datos experimentales a este tipo de modelo debido a que los parámetros de sorción de Langmuir se comportan como indicadores sensibles de la disponibilidad de P (Silva Rossi et al., 2013; Rollán y Bachmeier, 2014; Rashmi, Parama y Biswas, 2016; Silva Rossi et al., 2016).

Para las condiciones de este estudio, los valores de los cuadrados medios del error del modelo fueron bajos. Los gráficos de dispersión no presentaron tendencia alguna, lo que evidenció un adecuado ajuste de los datos experimentales al modelo seleccionado (Di Rienzo et al., 2013).

El ajuste coincide con el logrado por otros investigadores (Chimdi et al., 2013; Zamuner, Lloveras y Echeverría, 2015; Pose et al., 2016) quienes utilizaron el modelo de Langmuir en suelos neutros, no deficientes en $\mathrm{P}$, similares a los de este estudio. Por otra parte, difiere de lo observado por Vázquez y Morales (2000), Afif-Khouri (2007) y Al Salama (2008), quienes concluyen que el uso del modelo de Langmuir no es adecuado para describir el proceso de sorción de $\mathrm{P}$ para el rango de concentraciones medias y altas de $\mathrm{P}$ en solución (entre 60 y $640 \mathrm{mg} \mathrm{L}^{-1}$ ), como las que se aplican en suelos ácidos, ricos en arcilla, con alta capacidad de adsorción.

Tabla 2: Valores de los parámetros de sorción de la isoterma de Langmuir, cantidad máxima de fósforo adsorbido $\left(Q_{\max }\right)$ y constante de energía de retención ( $k$ ) de cada sitio y profundidad.

\begin{tabular}{ccccccc}
\hline Sitio & Profundidad $(\mathrm{cm})$ & $\boldsymbol{Q}_{\max }\left(\mathrm{mg} \mathrm{P} \mathrm{kg}^{-1}\right)$ & $\boldsymbol{k}\left(\mathrm{ml} \mathrm{g}^{-1}\right)$ & Profundidad $(\mathrm{cm})$ & $\boldsymbol{Q}_{\max }\left(\mathrm{mg} \mathrm{P} \mathrm{kg}^{-1}\right)$ & $\boldsymbol{k}\left(\mathrm{ml} \mathrm{g}^{-1}\right)$ \\
\hline 1 & $0-5$ & $368,75 \mathrm{a}^{2}$ & $0,42 \mathrm{a}^{2}$ & $0-20$ & $195,25 \mathrm{~b}^{1}$ & $0,62 \mathrm{~b}^{1}$ \\
2 & $0-5$ & $216,55 \mathrm{c}^{2}$ & $0,52 \mathrm{c}^{2}$ & $0-20$ & $182,80 \mathrm{~d}^{1}$ & $1,37 \mathrm{e}^{3}$ \\
3 & $0-5$ & $241,31 \mathrm{f}^{2}$ & $0,48 \mathrm{a}^{2}$ & $0-20$ & $152,40 \mathrm{~g}^{1}$ & $1,48 \mathrm{e}^{3}$ \\
4 & $0-5$ & $253,74 \mathrm{e}^{2}$ & $0,31 \mathrm{~d}^{2}$ & $0-20$ & $156,67 \mathrm{~g}^{1}$ & $0,91 \mathrm{f}^{1}$ \\
5 & $0-5$ & $242,87 \mathrm{f}^{2}$ & $0,52 \mathrm{c}^{2}$ & $0-20$ & $149,09 \mathrm{~h}^{1}$ & $1,67 \mathrm{e}^{1}$ \\
6 & $0-5$ & $222,88 \mathrm{c}^{2}$ & $0,37 \mathrm{~d}^{2}$ & $0-20$ & $130,46 \mathrm{~h}^{1}$ & $0,54 \mathrm{c}^{1}$ \\
\hline
\end{tabular}

Medias con distinta letra indican diferencias estadísticamente significativas $(p<0,001)$ en los datos de la fila. Medias con distintos superíndices numéricos indican diferencias estadísticamente significativas $(p<0,001)$ en los datos de la columna. 
Shi et al. (2015) y Stutte et al. (2015) señalan que para generar estrategias de gestión, manejo y aplicación de fertilizantes fosforados es necesaria una mejor comprensión del ciclo de P a nivel de lote, bajo condiciones específicas de suelo y manejo cultural. Este concepto se aplicó en el momento de determinar el valor máximo de concentración (40 $m g \mathrm{P} \mathrm{L}^{-1}$ ), el que resultó equivalente a una dosis entre 8 y $10 \mathrm{~kg} P$ ha-1 que, a su vez, se corresponde con las dosis de fertilizantes fosforados que se aplican en la región (Silva Rossi et al., 2013). Esto permitió analizar la isoterma bajo la influencia de condiciones específicas agronómicas de uso del nutriente (Shi et al., 2015; Stutte et al., 2015).

Además, el valor de concentración utilizado estuvo incluido en el rango de los valores de ajuste al modelo (Kruse et al., 2015), para las condiciones de este estudio, y por debajo de los valores que Vázquez y Morales (2000), Afif-Khouri, (2007) y Al Salama (2008) citan como inadecuados para este tipo de isoterma.

Los bajos niveles de arcilla, la reacción del suelo (próxima a la neutralidad) y los niveles de $\mathrm{P}$ en los lotes evaluados $\geq 15,3 \mathrm{mg} \mathrm{kg}^{-1} \mathrm{P}$-Bray (Tabla 1 ), homogenizaron el comportamiento de la matriz adsorbente en el espesor de 0 a $20 \mathrm{~cm}$ de profundidad, enmascarando las diferencias edáficas generadas por la SD en los primeros centímetros de profundidad del horizonte A. Calviño et al. (2000) y Picone, Capozzi, Zamuner, Echeverría y Sainz Rozas (2007) explican esta "homogenización", basados en que algunos de los cambios atribuidos a la SD producen efectos opuestos sobre la disponibilidad de P. Así, la suma de efectos en gran parte se anula, al integrar igual volumen de suelo en la profundidad 0 a $20 \mathrm{~cm}$, lo que explica la falta de diferencias significativas entre sitios entre los valores de $Q_{\max }$ estimados por el modelo de 0 a $20 \mathrm{~cm}$ de profundidad.

Los valores de $Q_{\max }$ (Tabla 2) son significativamente menores a los reportados por Vázquez y Morales (2000) para suelos de lotes de cultivo con altos contenidos de arcilla y aluminio extraíble y superan a los informados por Al Salama (2008), quien reporta $Q_{\max }$ entre 23 y $56 \mathrm{mg} \mathrm{P} \mathrm{kg}^{-1}$ correspondiendo el valor más alto a un suelo calcáreo y el más bajo a un suelo arenoso. Estas diferencias destacan, tal como lo señalan Shi et al. (2015) y Stutte et al. (2015), la importancia que tiene la comprensión del ciclo del P en el suelo bajo condiciones ambientales específicas.

La mayor amplitud en el rango de adsorción máxima entre $S$ se observó a nivel superficial. El $Q_{\max }$ varió entre 223 y $369 \mathrm{mg} \mathrm{P} \mathrm{kg}^{-1}$ de 0 a $5 \mathrm{~cm}$ y de 130 a $195 \mathrm{mg} \mathrm{P} \mathrm{kg}^{-1}$ de 0 a $20 \mathrm{~cm}$.
A diferencia de lo detectado entre sitios, los parámetros estimados para cada profundidad dentro de cada uno de ellos difirieron significativamente entre sí $(p<0,001)$. Los valores de $Q_{\max }$ de 0 a 5 $\mathrm{cm}$ fueron hasta $42 \%$ mayores a los de 0 a $20 \mathrm{~cm}$. Mientras que los valores de $k$ de 0 a $5 \mathrm{~cm}$ fueron significativamente $(p<0,001)$ menores a los de las muestras de 0 a $20 \mathrm{~cm}$ (Tabla 2).

Los cambios físicos, químicos y funcionales generados sobre la matriz del suelo por la SD continua en los primeros centímetros de profundidad (Rollán y Bachmeier, 2015) explican las diferencias observadas entre las profundidades de un mismo S. Así, gran parte de la variación en la magnitud de los parámetros de sorción de los primeros centímetros de suelo se debe a la acumulación diferencial de COS en dicho espesor (Tabla 1), una característica común en lotes bajo SD continua (Álvaro Fuentes et al., 2008; Álvarez et al., 2011).

Los altos contenidos de COS aportaron una mayor cantidad de sitios de adsorción de fosfatos, razón por la cual, a muy bajas concentraciones de $\mathrm{P}$ en solución, la adsorción ajustó a una isoterma casi lineal (Von Wandruszka, 2006), como se muestra a modo de ejemplo en la Figura 1 para el Sitio 1. Esto se debe a que los fenómenos de sorción a nivel superficial ocurren principalmente por interacción de los iones fosfato con las especies orgánicas presentes, dado que la fracción orgánica del suelo representa un medio activo de adsorción de $\mathrm{P}$ (Costa et al., 2017).

La diferencia porcentual entre los valores de $Q_{\max }$ de $0-5$ contra $0-20 \mathrm{~cm}$ indica que se necesita aplicar hasta un $42 \%$ más de $\mathrm{P}$ para saturar al estrato superficial en el nutriente, lo que coincide con lo observado por Rubio, Cabello y Gutierrez

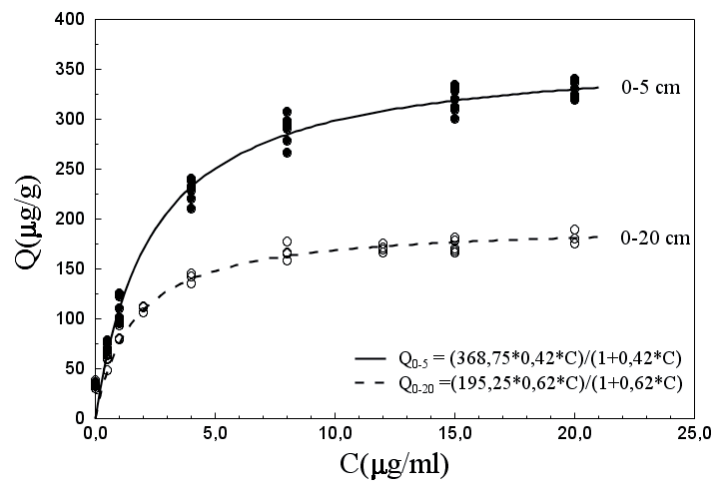

Figura 1: Relación entre el fósforo adsorbido $(Q)$ y la concentración de la solución en equilibrio $(\mathrm{C})$ para las profundidades 0-5 y 0-20 cm del Sitio 1. Las líneas continuas corresponden al modelo de Langmuir que ajusta los datos experimentales; en la leyenda se informan los parámetros para cada profundidad. 
Boem (2008), quienes determinaron que se necesita mayor cantidad de P para elevar el componente extractable del suelo cuanto mayor es el contenido de MOS.

Por otro lado, los mayores valores de $Q_{\max }$ a nivel superficial aumentan el valor residual de las aplicaciones de fertilizantes (Von Wandruszka, 2006; Pose et al., 2016), ya sea que se realicen aplicaciones al voleo o se incorpore el nutriente previo a la siembra (Picone et al., 2007; Ciampitti, Picone, Rubio y García, 2011; Gordon-Mendoza, FrancoBarrera, Villarreal-Núñez y Smith, 2016).

La mayor capacidad de adsorción de 0 a $5 \mathrm{~cm}$, asociada a bajos valores de la constante de energía, $k$, facilita el proceso de desorción de fosfatos hacia la solución del suelo en los primeros centímetros de profundidad, precisamente el espesor de colocación de los fertilizantes (Füleky y Tolner, 2006; Bravo et al., 2013).

El concepto del grado de saturación de P asociado a $Q_{\text {max }}$ se introdujo recientemente como un índice medioambiental de la cantidad de $\mathrm{P}$ que puede escurrir y lixiviar hacia las aguas superficiales o también subterráneas (AI Salama, 2008; White et al., 2014; Pose et al., 2016). Para los suelos bajo estudio, es posible inferir que los mayores valores de $Q_{\max }$ de 0 a $5 \mathrm{~cm}$ de profundidad contribuyen a reducir el impacto ambiental de la aplicación de fertilizantes fosforados.

\section{Relación entre los parámetros de adsorción y las propiedades edáficas}

Las características de la matriz del suelo determinan que la adsorción de P sea una propiedad intrínseca de cada individuo suelo, resultado de las reacciones de superficie que ocurren entre los iones fosfato y los constituyentes de dicha matriz (Chien, 2007).
La relación entre los parámetros de sorción y las propiedades del suelo se evaluó mediante coeficientes de correlación simple. El estudio mostró relaciones significativas $(p<0,001)$ entre las profundidades de muestreo y los valores $Q_{\max }$ y $k$.

La relación entre la cantidad de $\mathrm{P}$ adsorbido y la concentración de $\mathrm{P}$ en equilibrio también fue significativa $(p<0,01)$ con valores de $-0,68$ y $-0,91$ de $r^{2}$ para las profundidades de 0-20 y 0-5 cm, respectivamente. Brand-Klibanski, Liator y Shenker (2007) explican la importancia de esta relación señalando que, en suelos no arcillosos, la concentración de $P$ en equilibrio puede ser más importante que el $Q_{\max }$ en la predicción del riesgo ambiental del uso de fertilizantes fosforados. Este concepto se aplica a los suelos bajo estudio con bajos valores de $Q_{\max }$ (<600 mg P/kg), a partir de los cuales se puede provocar la eutrofización de las aguas dulces debido al uso intensivo de fertilizantes, expuestos a un inadecuado manejo nutricional (White et al., 2014).

Los coeficientes de correlación entre las propiedades edáficas (COS, pH, $\mathrm{ClC}, \mathrm{Ca}^{2+}{ }_{i n t}$ ) y los parámetros de adsorción se presentan en la Tabla 3. El coeficiente de correlación entre el COS y $Q_{\max }$ brinda información del alto grado de asociación entre ambas variables $(r=0,94, p<0,05)$. Moazed et al. (2010) concluyen que el COS y el grado de saturación con $\mathrm{Ca}^{2+}$ y $\mathrm{Al}^{3+}$ intercambiables son los principales predictores del P-adsorbido.

En relación con el efecto del COS sobre la sorción de P, Hiradate y Uchida (2004) explican que esto se debe al rol de la MOS en ese proceso, cuya función consiste en mantener un adecuado número de sitios disponibles para adsorción del nutriente, sin afectar el grado de afinidad (k). Así se observa en este estudio, en el que los mayores valores de $Q_{\max }(0-5 \mathrm{~cm})$ se asocian a bajos valores de la constante $k$. Bravo et al. (2013) señalan que esta relación es una prueba del efecto protector de

Tabla 3: Relación entre las propiedades edáficas: carbono orgánico (COS), $\mathrm{pH} 1: 2,5$, calcio intercambiable $\left(\mathrm{Ca}^{+2}\right)$, capacidad de intercambio catiónico $(\mathrm{CIC})$ y los parámetros de sorción $\left(Q_{\max }\right.$ y k) para cada una de las profundidades estudiadas $(0-5$ y 0-20 cm).

\begin{tabular}{lccccc}
\hline \multirow{2}{*}{ Propiedad } & Profundidad & \multicolumn{2}{c}{$Q_{\max }$} & \multicolumn{2}{c}{$k$} \\
\cline { 2 - 5 } COS & $(\mathrm{cm})$ & $\mathrm{r}^{2}$ & $\mathrm{p}$-valor & $\mathrm{r}^{2}$ & $\mathrm{p}$-valor \\
& $0-5$ & $-0,12$ & 0,818 & 0,94 & 0,005 \\
Arcilla & $0-20$ & $-0,35$ & 0,498 & 0,06 & 0,915 \\
& $0-5$ & 0,53 & 0,275 & 0,03 & 0,944 \\
$\mathrm{pH}$ & $0-20$ & 0,86 & 0,029 & 0,65 & 0,165 \\
& $0-5$ & 0,56 & 0,275 & 0,71 & 0,112 \\
$\mathrm{Ca}^{+2}$ & $0-20$ & 0,29 & 0,574 & 0,39 & 0,449 \\
& $0-5$ & 0,50 & 0,246 & 0,15 & 0,772 \\
$\mathrm{CIC}$ & $0-20$ & 0,10 & 0,845 & 0,44 & 0,380 \\
& $0-5$ & 0,54 & 0,271 & 0,32 & 0,526 \\
\hline
\end{tabular}


la MOS sobre la retención fosfórica y contribuye al incremento en forma paulatina de la disponibilidad de fósforo para los cultivos.

McLaughlin et al. (2011) señalan que no en todos los casos se encuentra una relación tan directa entre la MOS y la adsorción de P. Estos autores explican que en los sistemas bajo cultivo el P se acumula casi predominantemente en forma inorgánica en el suelo, asociado con aluminio, hierro y calcio intercambiables, según el tipo de suelo; en este sentido coinciden con Moazed et al. (2010), Tejada-Tovar, Villabona-Ortiz y Garcés-Jaraba (2015), Rosolem y Merlin (2014), entre otros, a la vez que atribuyen un alto valor predictivo del $Q_{\max }$ para definir el tipo de catión que satura el complejo de cambio. En los suelos bajo estudio el $\mathrm{Ca}^{2+}$ fue el catión dominante (Tabla 1). Los contenidos medidos de 0 a 5 y 0 a $20 \mathrm{~cm}$ de profundidad representaron entre el $64 \%$ y $68 \%$ del valor de la CIC, respectivamente. Sin embargo, y a diferencia de lo observado por Tejeda-Tovar et al. (2015), el P adsorbido no guardó relación con dichos contenidos en $\mathrm{Ca}^{2+}$ intercambiable. Rosolem y Merlin (2014) explican que los valores de sorción son más altos y, en consecuencia, es mayor la cantidad de $\mathrm{P}$ inorgánico unido a $\mathrm{Ca}^{2+}$ cuando se utilizan fosfatos naturales (compost o residuos orgánicos), observándose un comportamiento dispar con las fuentes de P inorgánicas, como las utilizadas en este estudio.

En ninguna de las profundidades evaluadas hubo correlación significativa entre los parámetros de sorción y las características edáficas con excepción del COS (0-5 cm). Vazquez y Morales (2000) observaron el mismo comportamiento; estos autores concluyen que esta falta de correlación indica que ninguno de los parámetros edáficos por sí solo puede explicar la capacidad máxima de sorción del suelo hacia el $\mathrm{P}\left(Q_{\max }\right)$. En tal sentido, Pinto et al. (2013) indican que esa falta de correlación pone en evidencia dos aspectos: a) por un lado, utilizar un único atributo del suelo (contenido de arcilla) como criterio para la recomendación de la fertilización fosfatada, como se hace rutinariamente, es susceptible a errores; y b) por otro, que, en función de la complejidad de las interacciones involucradas en el proceso de sorción, se deben continuar los estudios predictivos con el uso del mayor número posible de atributos o propiedades edáficas para lograr una recomendación más precisa.

Frente a la complejidad de las interacciones entre el $\mathrm{P}$ aplicado y la matriz del suelo Haggard y Sharpley (2006), señalan la importancia de realizar a futuro estudios que permitan conocer los procesos in-stream de $\mathrm{P}$, como una sucesión o flujo de fenómenos que incluyen sorción y desorción, precipitación y disolución, captación microbiana y retención, etc., los cuales ocurren en forma simultánea.

\section{CONCLUSIONES}

En condiciones de SD, la estratificación de los contenidos de COS genera un comportamiento diferencial de la matriz adsorbente, lo que debe tenerse en cuenta al definir la profundidad de muestreo con fines diagnóstico de la fertilidad edáfica en fósforo. Es de esperar que la mayor capacidad de adsorción $\left(Q_{\max }\right)$ y la baja afinidad de retención, $k$, que presenta la matriz del suelo de 0 a $5 \mathrm{~cm}$ afecten la residualidad de los fertilizantes aplicados en ese espesor.

En los suelos bajo estudio, para el planteo de una fertilización estratégica, es necesario incluir con fines de diagnóstico la evaluación de la capacidad de sorción $\left(Q_{\max }\right)$ y el grado de afinidad matriz-nutriente $(k)$, ya que ambos parámetros regulan la dinámica del $\mathrm{P}$ aplicado.

\section{AGRADECIMIENTO}

Los autores agradecen a la Secretaría de Ciencia y Tecnología de la Universidad Nacional de Córdoba por el apoyo económico recibido.

\section{BIBLIOGRAFÍA}

Afif-Khouri, E. y Oliveira, J. A. (2007). Agronomic efficiency of three phosphorus sources on grass turf under controlled conditions. Pastos, XXXVIII(1),45-63.

Al Salama, Y. J. (2008). Added Behavior of Different Phosphorus to Soil types Deir Ezzor Governorate, in Syria. Tishreen University Journal for Scientific Research and Studies. Biological Sciences Series, 30(5), 193-201.

Álvarez, C. R., Costantini, A. O., Bono, A., Taboada, M. A., Gutierrez-Boem, F. H., Fernández, P. L., y Prystupa, P. (2011). Distribution and vertical stratification of carbon and nitrogen in soil under different managements in the Pampean Region of Argentina. Revista Brasileira de Ciencia do Solo, 35,1985-1994.

Álvaro Fuentes, A. J., López Sánchez, M. V., Cantero-Martínez, C., y Arrúe Ugarte, J. L. (2008). Tillage effects on soil organic carbon fractions in Mediterranean dryland agroecosystems. Soil 
Science Society of America Journal, 72(2), 541547.

Brand-Klibanski, S., Liator, M. I., y Shenker, M. (2007). Overestimation of phosphorus adsorption capacity in reduced soils: An artifact of typical adsorption experiments. Soil Science Society of America Journal, 71,1128-1136.

Bravo, I., Montoya, J. C., y Menjivar, J. C. (2013). Retención y disponibilidad de fósforo asociado a la materia orgánica en un Typic Melanudands del departamento del Cauca, Colombia Acta Agronómica, 62(3), 261- 267.

Calviño, P., Echeverria, H. E., y Redolatti, M. (2000). Estratificación de fósforo en el suelo y diagnóstico de la fertilización fosfatada en trigo en siembra directa. XVII Congreso Argentino Ciencia del Suelo. Mar del Plata, Buenos Aires, Argentina. Actas en CD.

Carreira, J. A., Viñegla, B., y Lajtha, K. (2006). Secondary $\mathrm{CaCO}_{3}$ and precipitation of P-Ca compounds control the retention of soil $P$ in arid ecosystems. Journal of Arid Environments, 64(3), 460-473.

Chien, S. H. (2007). Análisis del suelo para la aplicación de las rocas fosfóricas. En Zapata F. y Roy, F. N. (Eds.). Utilización de las rocas fosfóricas para una agricultura sostenible, Capítulo 6, p. 6372. Roma, FAO.

Chimdi, A., Gebrekidan, H., Tadesse, A. y Kibret, K. (2013). Phosphorus Sorption Patterns of Soils from Different Land Use Systems of East Wollega, Ethiopia. American-Eurasian Journal of Scientific Research, 8(3), 109-116.

Ciampitti, I. A., Picone, L. I., Rubio, G., y García, F. O. (2011). Pathways of Phosphorous Fraction Dynamics in Field Crop Rotations of the Pampas of Argentina. Soil Science Society of America Journal, 75, 918-926.

Costa, A. R., Silva, J., Lopes Kern, M., Ruivo, C. D., Pinheiro, M. de L. y Marichal, R. (2017). Forms of soil organic phosphorus at black earth sites in the Eastern Amazon. Revista Ciência Agronômica, 48(1), 1-12.

Di Rienzo, J. A., Casanoves, F., Balzarini, M. G, González, L., Tablada, M., y Robledo, C. W. (2013). InfoStat, versión 2013, Grupo InfoStat, FCA, Universidad Nacional de Córdoba, Argentina. Recuperado el 18/12/2016 de http://www.infostat.com.ar

Eriksson, A. K. (2016). Phosphorus speciation in Swedish agricultural clay soils. Influence of fertilization and mineralogy. Doctoral Thesis. Swedish University of Agricultural Sciences, Uppsala. Recuperado de: https://pub.epsilon.slu.se/13057/1/ eriksson_ak_160212.pdf
Fernández López, C., y Mendoza, R. (2013). Added phosphorus availability and re-distribution in both natural and cultivated vertisols. Ciencia del Suelo, 31(2), 143-152.

Füleky, G. y Tolner, L. (2006). Determination of the phosphate content originally adsorbed on the soil by fitting an adsorption isotherm model. Applied Ecology and Environmental Research, 4(2), 39-45.

Ghida Daza, C. y Sánchez, C. (2009). Zonas Agroeconómicas Homogéneas: Córdoba. Estudios socioeconómicos de la sustentabilidad de los sistemas de producción y recursos naturales N ${ }^{\circ} 10$. Ciudad Autónoma de Buenos Aires: Ediciones INTA.

Gordon-Mendoza, R., Franco-Barrera, J. E., ViIlarreal-Núñez, J. E., y Smith, T. J. (2016). Manejo de la fertilización fosforada en el cultivo de maíz, el Ejido, Panamá 2004-2013. Agronomía Mesoaméricana, 27(1), 95-108.

Haggard, B. E. y Sharpley, A. N. (2006). Phosphorus transport in streams: Processes and modeling considerations. En Radcliffe, D. E. y Cabrera, M.L. (Eds.), Modeling phosphorus in the environment. Boca Raton, FL.: CRC Press, p. 105-130.

Hiradate, S. y Uchida, N. (2004). Effects of soil organic matter on $\mathrm{pH}$-dependent phosphate sorption by soils, Soil Science and Plant Nutrition, 50(5), 665-675.

Instituto Nacional de Tecnología Agropecuaria (2006). Recursos naturales de la provincia de Córdoba. Los suelos, nivel de reconocimiento 1:500.000. Córdoba, Argentina: Agencia Córdoba Ambiente-Instituto Nacional de Tecnología Agropecuaria.

Kruse, J. M., Abraham, M., Amelung, W., Baum, C., Bol R., Kühn, O., Lewandowski, H., Niederberger, J., Oelmann, Y., Rüger, C., Santner, J., Siebers, M., Spohn, M., Vestergren, J., Vogts, A., y Leinweber, P. (2015). Innovative methods in soil phosphorus research: A review. Journal of Plant Nutrition and Soil Science, 178, 43-88.

Martínez, H. E., Fuentes, J. P. y Acevedo H. E. (2008). Carbono orgánico y propiedades del suelo. Revista de la ciencia del suelo y nutrición vegetal, 8(1), 68-96

McLaughlin M. J., McBeath, T. M., Smernik, R., Stacey, S. P., Ajiboye, B. y Guppy, C. (2011). The chemical nature of $P$ accumulation in agricultural soils - implications for fertilizer management and design: an Australian perspective. Plant and Soil, 349(1), 69-87.

Moazed, H., Hoseini, Y., Naseri, A. A. y Abbasi, F., (2010). Determining phosphorus adsorption isotherm in soil and its relation to soil characteris- 
tics. Journal of Food, Agriculture \& Environment, 8(2), 1153-1157.

Moreira, W. H., Tormena, C. A., Karlen, D. L., Pires da Silva, A., Keller, T. y Betiol, E. (2016). Seasonal changes in soil physical properties under longterm no-tillage. Soil \& Tillage Research, 160, 53-64.

Nelson, D. W. y Sommers, L. E. (1996). Total carbon, organic carbon, and organic matter. En Sparks D. L. (Ed.), Methods of Soil Analysis. Part 3, Madison WI: ASA, SSSA, pp. 961-1010.

Picone, L., Capozzi, I., Zamuner, E., Echeverría, H. y Sainz Rozas, H. (2007). Transformaciones de fósforo en un molisol bajo sistemas de labranza contrastantes. Ciencia del Suelo, 25(2), 99-107.

Pinto, F. A., Damacena de Souza, E., Barbosa Paulino, H., Curi, N. y Carbone Carneiero, M. A. (2013). P-Sorption and desorption in Savanna Brazilian soils as a support for phosphorus fertilizer management. Ciência e Agrotecnologia, 37(6), 521-530.

Pose, N. N., Zamuner, E. C., Picone, L. I., Videla, C. C., Rodríguez, S. y Maceira, N. (2014). Evaluación de indicadores de retención de fósforo en sistemas agrícolas, ganaderos y forestales. Chilean Journal of Agricultura Land Animal Science, ex Agro-Ciencia, 30(1), 65-73.

Pose, N. N., Baeza, M. C., Zamuner, E. C., Di Gerónimo, P. y Videla C. del C. (2016). Parámetros agronómicos y ambientales de fósforo en suelos molisoles con diferentes usos en la provincia de Buenos Aires, Argentina. Acta Agronómica, 65(4), 375-382.

Rashmi, I., Parama, V. R. R. y Biswas, A. K. (2016). Phosphate sorption parameters in relation to soil properties in some major agricultural soils of India. SAARC J. Agri., 14(1), 1-9.

Richardson, A. E., Condron, L. M. y Haygarth, P. M. (2015). Land use and soil factors affecting accumulation of phosphorus species in temperate soils. Geoderma, 257-258, 29-39.

Rollán, A. A. del C. y Bachmeier, O. A. (2014). Diffusional transport of chloride and phosphate in soils of the North Central Region of Córdoba (Argentina). IJRDET, 3(4), 62-65.

(2015). Effect of Continuous Zero Tillage on the Physical-Functional Behavior of Silty Loam Soils of the Semiarid Central Region of the Province of Córdoba (Argentina). Terra Latinoamericana, 33(4), 275-284.

Rollán, A. A. del C. (2016). Efecto de la Siembra Directa Continua en Suelos de Córdoba, Argentina. Estudios Regionales. Saarbrücken (Alemania), Editorial Académica Española.
Ron, M. de las M., Mandolesi, M. E., Facchinetti, C. y Jürgen Kiessling, R. (2011). Efecto antrópico sobre la fertilidad química de un suelo en el sudoeste bonaerense. Ciencia del suelo, 29(2), 223231.

Rosolem, C. A. y Merlin, A. (2014). Soil phosphorus availability and soybean response to phosphorus starter fertilizer. Revista Brasileira de Ciência do Solo, 38(5), 1487-1495.

Rubio, G., Cabello, M. J. y Gutiérrez Boem, F.H. (2008). Estimating available soil phosphorus increases after phosphorus additions in Mollisols. Soil Science Society of America Journal. 72, 17211727.

Sainz Rozas, H. R., Echeverria, H. E. y Angelini, H. P. (2011). Niveles de carbono orgánico y pH en suelos agrícolas de las regiones pampeana y extrapampeana argentina. Ciencia del suelo, 29(1), 29-37.

(2012). Fósforo disponible en suelos agrícolas de la región Pampeana y Extra Pampeana Argentina. RIA, 38(1), 33-39.

Sánchez, C. y Barberis, N. A. (2013). Caracterización del Territorio Centro de la provincia de Córdoba. Manfredi, Córdoba. Ediciones INTA.

Schmidt, E. S. y Amiotti, N. M. (2015). Propiedades edáficas superficiales en sistemas de agricultura de conservación en la región pampeana semiárida sur. Ciencia del suelo, 33(1), 79-88.

Shi, Y., Ziadi, N., Messiga, A. J., Lalande, R. y $\mathrm{Hu}, \mathrm{Z}$. (2015). Soil phosphorus fractions change in winter in a corn-soybean rotation with tillage and phosphorus fertilization. Pedosphere, 25(1), 1-11.

Silva Rossi M. M., Rollán, A. A. del C. y Bachmeier, O. A. (2013). Phosphorus availability in the central area of the Argentine Pampean region. 1: Relationship between soil parameters, adsorption processes and wheat, soybean and corn yields in different soil and management environments. Spanish Journal of Soil Science, 3(1), 45-47.

(2016). Available phosphorus in the central area of the argentinean pampas. 2: Kinetics of adsorption and desorption of phosphorus under different soil and management environments. Spanish Journal of Soil Science, 6(2), 145-158.

Soil Conservation Service. (1972). Soil survey laboratory methods and procedures for collecting soils samples. Soil Survey manual. Report, 1. USDA, Washington (USA).

Sparks, D. (1995). Environmental Soil Chemistry. Chapter 5: Sorption phenomena in soil. San Diego, USA. Academic Press.

Stutte, M. I., Shand, C. A., George T. S., Blac- 
kwell, M. S. A., Dixon, L., Bol, R., MacKay, R. L., Richardson, A. E., Condron, L. M. y Haygarth, P. M. (2015). Land use and soil factors affecting accumulation of phosphorus species in temperate soils. Geoderma, 257-258, 29-39.

Tejada-Tovar, C., Villabona-Ortiz, A. y GarcésJaraba, L. (2015). Adsorción de metales pesados en aguas residuales usando materiales de origen biológico. Revista Tecno Lógicas, 18(34), 109-123.

Vázquez, S. y Morales, L. (2000). Adsorción de fósforo por suelos ácidos de Misiones (Argentina). Ciencia del Suelo, 18(2), 89-94.

Vázquez, S., Morales, L. A., Fernández López, C. y Dalurzo, H. C. (2011). Fertilización fosfatada y fracciones de fósforo en alfisoles, ultisoles y oxisoles. Ciencia del Suelo, 29(2), 161-171.

Von Wandruszka, R. (2006). Phosphorus retention in calcareous soils and the effect of organic matter on its mobility. Geochemical Transactions, 7(6), 1-8

White, M. J., Storm, D. E., Mittelstet, A., Busteed, P. R., Haggard, B. E. y Rossi, C. (2014). Development and Testing of an In-Stream Phosphorus Cycling Model for the Soil and Water Assessment Tool. Journal of Environmental Quality, 43(1), 215223.

Yadav, B. K. y Verma, A. (2012). Phosphate solubilization and mobilization in soil through soil microorganisms under arid ecosystems, the functioning of ecosystems. En Ali, M. (Ed.) In Tech., Recuperado de http://www.intechopen.com/books/ the-functioning-of-ecosystems/

Zamuner, E. C., Lloveras, J. y Echeverría, H. (2015). Métodos agronómicos y ambientales de determinación de fósforo en Argiudoles del sudeste bonaerense. Ciencia del Suelo, 33(1), 55-63. 